geht, ist die Zulässigkeit und Durchführung einer Abänderung des Wertausgleichs bei der Scheidung in den $\mathbb{S} \mathbb{S} 225$ und 226 FamFG-E geregelt.

Danach findet eine Überprüfung nur statt, soweit die Rechte im Sinne des $\mathbb{3} 32$ VAStrRefG betroffen sind. Die Wertänderung muss zudem wesentlich sein, wobei nach Maßgabe der Entwurfsbegründung $\mathbb{S} 323 \mathrm{ZPO}^{8}$ heranzuziehen ist.

In diesem Zusammenhang darf jedoch nicht unerwähnt bleiben, dass eine „Fehlerkorrektur“ der Anrechte aus der 2. und 3. Säule ${ }^{9}$ nicht mehr möglich ist.

Die dagegen vorgebrachte Kritik ${ }^{10}$ ist durchaus berechtigt. Denn der Grundsatz der „gerechten Halbteilung “ wird (erneut) in Frage gestellt (unabhängig von der Verfassungsmäßigkeit der Regelung).

Ein weiterer (Kritik-)Punkt ist die Anpassung wegen Unterhalts. ${ }^{11}$ Die Vorschriften entsprechen dem $\mathbb{S}$ VAHRG mit dem - entscheidenden - Unterschied, dass die Korrektur nicht mehr durch den Leistungsträger des Verpflichteten erfolgt, sondern durch das Familiengericht ${ }^{12}$. Die sich abzeichnende Mehrbelastung der Gerichte ist bereits Gegenstand der Diskussion. ${ }^{13}$

\section{Ausblick}

Das (gesetzgeberische) Vorhaben einer umfassenden Reform des Versorgungsausgleichs ist - im Ergebnis - zu begrüßen. Denn die Alternative besteht allein in der Beibehaltung des bisherigen Systems mit versicherungsmathematischer „Nachberechnung “ (Modell Bergner).

Wünschenswert wäre jedoch eine Nachbesserung des Entwurfs aufgrund der im Detail geäußerten Vorbehalte gegenüber Einzelnormen des Entwurfs. Dies gilt auch für den Ausgleich der Anrechte aus der Beamtenversorgung. Denn die (nunmehr) für die Landesbediensteten zuständigen Bundesländer haben gegenüber dem Grundsatz der internen Teilung verfassungsrechtliche Bedenken (Grundsatz der Alimentation) erhoben, die durch $\$ 16$ VAStrRefG nicht als ausgeräumt angesehen werden. Um hier einen „Flickenteppich“ zu vermeiden, sollte (rechtzeitig) über eine einvernehmliche Lösung nachgedacht werden.

\footnotetext{
$8 \S 238$ FamFG-E.

9 Betriebliche Altersversorgung, Lebensversicherung auf Rentenbasis.

10 Born a.a.O. S. 2292 unter Hinweis auf Rehme FamRZ 08, 738 ff.

$11 \S \S 33,34$ VAStrRefG.

§ 34 Abs. 1 VAStrRefG.

Born a.a.O. S. 2293
}

\title{
Das Kind im internationalen Familienrecht - ein kurzer Überblick
}

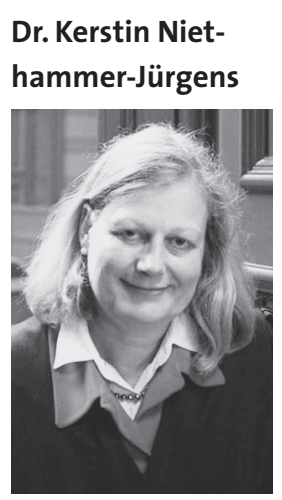

Mitglied der Kommission Zivil-, Familienund Erbrecht, Recht anderer Lebensgemeinschaften des djb, Rechtsanwältin und Fachanwältin für Familienrecht, Potsdam
Die anwaltliche familienrechtliche Beratung sieht sich einer zunehmenden Binationalität der Mandantschaft sowie einer wachsenden Zahl von Familien gegenüber, in denen eines oder mehrere Mitglieder nicht die deutsche Staatsangehörigkeit besitzen oder nicht in Deutschland leben. Ca. 700.000 deutsche Staatsangehörige haben einen ausländischen Ehepartner, allein an jeder sechsten in Deutschland geschlossenen Ehe ist ein nicht deutscher Partner beteiligt, wobei nur 2,7 Prozent dieser Eheschließungen ausschließlich unter Ausländern erfolgen. 2,5 Millionen minderjährige Kinder leben darüber hinaus in Deutschland, bei denen zumindest ein Elternteil nicht die deutsche Staatsangehörigkeit besitzt.

Nachdem die Ehen mit ausschließlicher Ausländerbeteiligung sowie die gemischt nationalen Ehen in ihrer Scheidungshäufigkeit den „rein deutschen “ Ehen in nichts nachstehen, liegt auch hier die Scheidungsrate bei knapp 45 Prozent.

Die Interessen der Kinder, die in einen Scheidungs- oder Trennungsprozess mit Auslandsbezug involviert sind, müssen in der innerfamiliären Auseinandersetzung besonders geschützt werden. Hierzu steht ein Normgefüge zur Verfügung, das insbesondere dem in Art. 8 der Europäischen Menschenrechtskonvention und Art. 6 GG festgeschriebenen
Schutz der Familie dient. Die internationalen Übereinkommen und EU-Verordnungen unterliegen damit der Anforderung an jede familiengerichtliche Entscheidung, diese zum Wohl und im Sinne des Kindes zu treffen. Dasjenige, was wir im deutschen Recht mit Kindeswohl umschreiben, durchzieht die Übereinkommen und Verordnungen, wobei allerdings die Ausgangs- und Schwerpunkte der insoweit einschlägigen Normen unterschiedlich und dabei ausgerichtet sind am Ziel der jeweiligen Norm.

\section{Brüssel Ila-VO (Eheverordnung)}

Soweit es um den Schutz des Kindes in Umgangsund Sorgerechtsverfahren geht, ist für Mitgliedstaaten der Europäischen Gemeinschaft (EU) mit Ausnahme Dänemarks seit dem 1. März 2005 auch für die neuen Mitgliedstaaten der EG nunmehr einschlägig die Eheverordnung ${ }^{1}$ (EheVO, auch Brüssel IIaVO genannt).

Die EheVO, die ihre Vorgängerverordnung bereits nach drei Jahren abgelöst hat, hat die Beschränkung

\footnotetext{
1 Verordnung (EG) Nr. 2201/2003 des Rates über die Zuständigkeit und die Anerkennung und Vollstreckung von Entscheidungen in Ehesachen und in Verfahren betreffend die elterliche Verantwortung und zur Aufhebung der Vorgängerverordnung (EG Nr. 1347/2000).
} 
auf die ehegemeinschaftlichen Kinder aufgehoben. Der Regelungsbereich ergibt sich aus Art. 8 EheVO und bezieht sich auf alle Entscheidungen, die die elterliche Verantwortung - ein Begriff, den nur das europäische Familienrecht kennt - betreffen. Darunter fallen sämtliche Verfahren hinsichtlich der Personenoder Vermögenssorge Minderjähriger, insbesondere auch dann, wenn sie nicht im Zusammenhang mit einer Ehesache stehen. Die Verordnung regelt die internationalen Zuständigkeiten für diese Verfahren in den Artikeln 8 bis 10, ergänzt durch Art. 11 Abs. 1 und 2 sowie durch Art. 13 und insbesondere Art. 12 . Während Art. 8 eine Grundsatzanknüpfung an den gewöhnlichen Aufenthalt des Kindes normiert, finden sich in den Artikel 8, 9, 10 und 12 spezielle Anknüpfungen für bestimmte Teilbereiche der elterlichen Verantwortung. Die Grundsatzanknüpfung des Art. 8 Abs. 1 der EheVO ist ausschließlich, weswegen die Staatsangehörigkeiten des Kindes und der Eltern in diesem Zusammenhang keine Rolle spielen. Dies bedeutet, dass selbst bei übereinstimmender Staatsangehörigkeit aller am Verfahren Beteiligter der gemeinsame Heimatstaat nicht zuständig sein kann, wenn das Kind seinen gewöhnlichen Aufenthalt in einem anderen Mitgliedstaat der EheVO hat.

Um internationale Kompetenzkonflikte bei gleichzeitiger Anhängigkeit (nicht Rechtshängigkeit!) auch im Bereich der elterlichen Verantwortung zu vermeiden, legt Art. 19 Abs. 2 und 3 EheVO das strikte zeitliche Prioritätsprinzip fest, nämlich der Vorrang des früher „angerufenen“ Gerichtes. Das später angerufene Gericht setzt das Verfahren aus, bis das zuerst angerufene Gericht über die $\mathrm{Zu}$ ständigkeit entschieden hat.

Neben den Zuständigkeitsregelungen normiert Art. 21 Abs. 1 EheVO die Anerkennung einmal getroffener Entscheidungen. Eine einmal im Ausland ergangene Entscheidung zur elterlichen Sorge, zu Teilbereichen der elterlichen Sorge oder zum Umgang ist im Inland mit gleicher Wirkung wie eine entsprechende inländische Entscheidung anzuerkennen, wobei es eines separaten Anerkennungsund Vollstreckungsverfahrens nicht mehr bedarf, Art. 21 Abs. 1 EheVO.

Da die EheVO nicht die Frage des anzuwendenden materiellen Rechts erfasst, ist hinsichtlich des materiellen Rechts auf Artikel 21 EGBGB zurückzugreifen. Allerdings wird hier ebenfalls auf den gewöhnlichen Aufenthalt des Kindes abgestellt bzw. ist auf das $\mathrm{MSA}^{2}$ zurückzugreifen. Damit besteht der notwendige Gleichklang zwischen der Frage der internationalen Zuständigkeit eines Gerichts, das sich nach dieser Zuständigkeit folgerichtig ergebenden Verfahrensrechts sowie des dann anwendbaren materiellen Rechts.
Die EheVO verdrängt wegen ihres weitergehenden Anwendungsbereiches das MSA. Es gilt nur noch im Verhältnis zur Türkei und der Schweiz und hat im Anwendungsbereich der EheVO seine zuständigkeitsbegründende Bedeutung verloren.

\section{Haager Übereinkommen über die zivilrechtlichen Aspekte internationaler Kindesentführung (HKÜ)}

In der Präambel des $\mathrm{HKÜ}^{3}$ wird bereits formuliert, was die Vertragsstaaten ${ }^{4}$ bei grenzüberschreitenden innerfamiliären Entziehungen für kindeswohlentsprechend erachten: Eine möglichst schnelle (,,sofortige“) Rückführung („Rückgabe“) des Kindes in seinen Heimatstaat.

Eine weitergehende Überprüfung durch die Gerichte des Zufluchtsstaates soll nur im Hinblick auf die im Übereinkommen selbst festgelegten Tatbestände stattfinden. Das HKÜ entzieht in den Kindesentziehungsfällen daher den Gerichten des Zufluchtsstaates die internationale Zuständigkeit für Sachentscheidungen über das Sorgerecht und belässt diese bei den Gerichten des Herkunftsstaates. Die Tatbestände, bei deren Vorliegen von einer Rückführung abgesehen werden kann, sind als Ausnahmetatbestände formuliert; die Vorausset-

\section{In der Präambel des HKÜ wird formuliert, was die Vertragsstaaten bei grenzüberschreitenden innerfami- liären Entziehungen für kindeswohlentsprechend erachten: Eine möglichst schnelle („sofortige“) Rück- führung („Rückgabe“) des Kindes in seinen Heimatstaat.}

zungen sind von dem entziehenden Elternteil zu beweisen. $\mathrm{Zu}$ den Ausnahmetatbeständen gehört der Vortrag, dass das Sorgerecht durch den zurückgebliebenen Elternteil nicht ausgeübt wurde, dass der zurückgebliebene Elternteil seine Zustimmung oder nachträgliche Genehmigung zum Verbleib des Kindes im Zufluchtsstaat erteilt hätte, dass eine Rückführung des Kindes mit schwerwiegenden Gefahren eines körperlichen oder seelischen Schadens für das Kind verbunden sei oder es auch durch die Rückführung in eine entsprechende zumutbare Lage gebracht wird. Auch der Widerstand des urteilsfähigen Kindes gegen eine Rückführung sowie die Verletzung der geltenden Grundwerte über den Schutz

2 Haager Übereinkommen über die Zuständigkeit der Behörden und das anzuwendende Recht auf dem Gebiet des Schutzes von Minderjährigen vom 5.10.1961.

3 Haager Übereinkommens über die zivilrechtlichen Aspekte internationaler Kindesentführung vom 25.10.1980.

4 Zum aktuellen Stand der Mitgliedstaaten www. bundesjustizamt.de. 
von Menschenrechten und Freiheiten im Herkunftsland gehören nach dem Übereinkommen zu den Tatbeständen, nach denen von einer Rückführung im Ausnahmefall abgesehen werden kann, vgl. Art. 13 und 20 HKÜ.

$\mathrm{Zu}$ den Ausnahmetatbeständen gibt es bei Entziehungen in die Bundesrepublik Deutschland durch die Instanzgerichte eine sehr differenzierte, am Einzelfall ausgerichtete Rechtsprechung. Die deutschen Gerichte sind sich zunehmend bewusst, dass sie im Rahmen dieser zu führenden summarischen Eilverfahren keine Sorgerechtsentscheidungen zu treffen haben, sie sind sich darüber bewusst, dass eine Kindeswohlüberprüfung nur anhand der vorgenannten Ausnahmetatbestände vorzunehmen ist und dass es hier nicht um eine Entscheidung hinsichtlich der elterlichen Sorge mit einer umfassenden Kindeswohlprüfung geht.

Im Laufe der Zeit wurde die Diskussion darüber

\section{Zu den Ausnahmetatbeständen gibt es bei Entziehungen in die Bundesrepublik Deutschland durch die Instanz- gerichte eine sehr differenzierte, am Einzelfall ausgerich- tete Rechtsprechung. Die deutschen Gerichte sind sich zunehmend bewusst, dass sie im Rahmen dieser zu führenden summarischen Eilverfahren keine Sorgerechts- entscheidungen zu treffen haben.}

geführt, inwieweit die Einschaltung von Jugendämtern als Berichterstatter über die hiesigen Lebensverhältnisse des Kindes, die Anhörung des Kindes in dem Verfahren, die Bestellung von Verfahrenspflegern sowie die Erstellung zeitintensiver Gutachten auch in HKÜ-Verfahren notwendig und geboten sind. Auch hierzu gibt es inzwischen Einzelrechtsprechung, die nur in Ausnahmefällen die Einholung zeitintensiver Sachverständigengutachten zulässt, die Bestellung von Verfahrenspflegern aber für notwendig erachtet. Gerichtliche Anregungen an die Parteien im Hinblick auf eine Mediation im laufenden Verfahren werden allerdings zunehmend gefordert.

Bei all diesen Diskussionen wird immer darauf hinzuweisen sein, dass es sich beim dem HKÜ, dem mittlerweile über 70 Staaten beigetreten sind, zwar um einen völkerrechtlichen Vertrag handelt, dass dieses Übereinkommen aber letztendlich nach wie vor ein Rechtshilfeübereinkommen ist.

In den EU-Staaten - außer in Dänemark - hat allerdings die EheVO das HKÜ modifiziert. Bei widerrechtlichem Verbringen eines Kindes von einem Mitgliedstaat in einen anderen sowie bei dem $\mathrm{Zu}-$ rückhalten des Kindes in einem anderen Mitgliedstaat, dem klassischen Fall einer innerfamiliären Kindesentziehung, bleibt es zunächst bei der internationalen Zuständigkeit des Herkunftsmitgliedstaates. Soweit das Kind in dem Mitgliedstaat, in dem es sich nach einer Entziehung befindet, seinen gewöhnlichen Aufenthalt erlangt hat, endet die $\mathrm{Zu}$ ständigkeit des Herkunftsmitgliedstaates, wenn der neue Sorgeinhaber dem neuen Aufenthalt zugestimmt hat. Die Zuständigkeit endet darüber hinaus auch ohne Zustimmung mit Zeitablauf, nämlich dann, wenn der zurückgebliebene Elternteil seit einem Jahr Kenntnis von dem neuen Aufenthalt des Kindes hatte oder diese hätte haben müssen und während dieser Zeit keinen Rückführungsantrag oder ein Verfahren in seinem Herkunftsstaat eingeleitet hat.

Sofern das Gericht des Zufluchtsstaates des Kindes meint, dass der Ausnahmetatbestand des Art. 13 Abs. 1 b des HKÜ in Betracht kommt, d.h. dass dem Kind bei einer Rückführung schwerwiegende Gefahren für seine körperliche und seelische Gesundheit drohen, darf das Gericht die Rückgabe nicht verweigern, wenn ihm nachgewiesen wurde, dass im Herkunftsstaat des Kindes angemessene Vorkehrungen zu seinem Schutz getroffen wurden. In diesem Fall muss es seine Entscheidung innerhalb eines Monates an das eigentlich zuständige Heimatgericht des Kindes übermitteln, um den Eltern die Möglichkeit zu geben, innerhalb von drei Monaten dort eine Sorgerechtsentscheidung herbeizuführen. Wird diese dann zugunsten des zurückgebliebenen Elternteils getroffen, verbunden mit einer Rückgabeanordnung, hat diese Vorrang vor der im Zufluchtsstaat getroffenen Entscheidung und setzt sich damit gegen die im Zufluchtsstaat getroffene Entscheidung durch.

Eine weitere Modifizierung des HKÜ in der EheVO findet sich dadurch, dass die Anhörung des Kindes sichergestellt werden muss, sofern es aufgrund des Alters und des Reifegrades des Kindes nicht angebracht erscheint.

In der Praxis erfordern diese Modifizierungen des Haager Kindesentziehungseinkommens durch die EheVO eine Kooperation und Kommunikation der hiesigen Gerichte mit den Gerichten des Herkunftsstaates.

\section{Internationales Familienrechtsverfahrensgesetz (IntFamRVG)}

Nicht zuletzt um diese Kommunikation zu unterstützen, aber auch im Übrigen die EheVO und das HKÜ verfahrensrechtlich umzusetzen, gibt es im Anwendungsbereich der EheVO und des HKÜ seit dem 1. März 2005 ein neues internationales Ver- 
fahrensrecht. Das IntFamRVG 5 regelt neben den Aufgaben der deutschen Zentralen Behörde (Bundesamt für Justiz, Bonn) und des Jugendamtes das Rechtsmittelrecht und Vollstreckungsrecht, insbesondere aber die sachliche und örtliche gerichtliche Zuständigkeit des HKÜ-Verfahrens. Die Verfahren sind zentriert zu führen, die sachliche und örtliche Zuständigkeit liegt ausschließlich bei den Amtsgerichten am Sitz eines OLG.

Für die Praxis bedeutsam sind die auf Art. 39 EheVO beruhenden vereinfachten Verfahren auf Anerkennung ausländischer Entscheidungen zur elterlichen Verantwortung im Inland bzw. auf Anerkennung deutscher Entscheidungen in diesem Bereich im Ausland. Unter Verwendung eines Formblattes ist eine Bescheinigung dem inländischen bzw. ausländischen Gericht vorzulegen. Eines förmlichen Anerkennungsverfahrens bedarf es nicht mehr. Soll eine deutsche Entscheidung zur elterlichen Verantwortung in einem anderen Mitgliedstaat (ausgenommen Dänemark) anerkannt werden, ist die Bescheinigung nach Art. 39 EheVO im Inland zu beantragen. Die Bescheinigung ist gemäß $\mathbb{S} \mathbb{S} 48 \mathrm{f}$. IntFamRVG beim Urkundsbeamten des Gerichts, das die Entscheidung getroffen hat, zu beantragen.

\section{Internationales Adoptionsübereinkommen (AdoptÜ)}

Das Adopt $\ddot{U}^{6}$ ist für die BRD am 1. März 2002 in Kraft getreten. Als völkerrechtlicher Vertrag hat es die Belange des Kindeswohls in grenzüberschreitenden Adoptionsfällen sicherzustellen, diese zu wahren und zu fördern und damit das Kind vor Entführung, Verkauf und Handel zu schützen. Diese Grundsätze befinden sich bereits in der EU-Kinderkonvention von 1989, der Deutschland beigetreten ist. Das Adopt ̈̈ enthält keine Regelung, die dem nach dem internationalen Privatrecht der Mitgliedstaaten anzuwendenden Recht vorgehen und greift damit nicht in das Kollisionsrecht der Vertragsstaaten ${ }^{7}$ ein. Art. 22 EGBGB bestimmt das Adoptionsstatut und damit das anzuwendende materielle Recht, je nachdem, ob die Adoption durch Verheiratete oder Unverheiratete erfolgt. Da Art. 22 EGBGB die gemeinsame Adoption durch nicht verheiratete Partner nicht vorsieht, scheidet nach deutschem internationalen Privatrecht damit auch eine internationale Adoption durch eine registrierte gleichgeschlechtliche Partnerschaft aus, nachdem Art. 17 b Abs. 4 i.V.m. $\$ 1741$ Abs. 2 S. 1 BGB dieses ausschließt.

Das AdoptÜ nennt zum Schutz der Kinder und zum Erreichen dieser vorgenannten in Art. 1 des Übereinkommens genannten Ziele in Art. 4 konkrete Maßstäbe, nach denen eine internationale Adoption von Minderjährigen, nicht Volljährigen, durch die Vertragsstaaten durchgeführt werden kann und welche Maßnahmen die Behörden des Heimatstaates zum Schutz des Kindes haben ergreifen müssen. Danach sind die Behörden des Heimatstaates des Kindes verpflichtet, Ermittlungen über die Lage des Kindes im Heimatstaat des Kindes einzuholen. Die Behörden müssen festgestellt haben, dass die Adoption ins Ausland eher dem Kindeswohl entspricht als das Aufwachsen im Inland. Die Behörden des Heimatstaates müs- sen die potentiellen Adoptiveltern beraten und über die Wirkungen einer Adoption unterrichtet haben, das Kind muss unter Berücksichtigung seines Alters beraten und über die Wirkung der Adoption unterrichtet worden sein und ggf. nach seinen Wünschen und Meinungen gefragt worden sein. Und dies ist möglicherweise der wichtigste Punkt - es muss durch Behörden des Heimatstaates des Kindes gewährleistet sein, dass die Zustimmung zur Adoption nicht durch eine Zahlung oder andere Gegenleistung herbeigeführt wurde.

Die Vorgaben dieses völkerrechtlichen Übereinkommens werden in Deutschland durch das AdoptionsübereinkommensAusführungsgesetz vom 5. November $2001^{8}$ umgesetzt.

Bedeutsam für die Praxis ist in diesem Zusammenhang insbesondere die festgelegte Zuständigkeitskonzentration der Adoptionsverfahren mit ausländischem Sachbezug bei den Vormundschaftsgerichten am Sitz eines OLG, wodurch wie in den Fällen internationaler Kindesentziehung die besondere Fachkompetenz der Richterschaft gestärkt werden soll. Bei der anwaltlichen Beratung potentieller deutscher Adoptiveltern im Hinblick auf eine Adoption eines ausländischen Kindes ist nunmehr zu berücksichtigen, dass für Kinder aus dem Ausland nur noch Adoptionsvermittlungsstellen zugelassen sind, die eine besondere Zulassung erhalten haben. Diese Stellen haben bei ausländischen Adoptionen ein Vermittlungsmonopol.

Das AdoptÜ i.V.m. mit dem Ausführungsgesetz enthält schließlich Regelungen für die gegenseitige Anerkennung von Adoptionsentscheidungen in den anderen Vertragsstaaten. In dem so genannten Aufnahmestaat, d.h. in dem Staat, in dem die Adoption anzuerkennen ist, ist es danach nicht möglich, materiell rechtlich noch einmal zu überprüfen, ob die Voraussetzungen für die Annahme gegeben waren oder nicht. Insoweit erforderlich ist lediglich eine Bescheinigung des Staates, in dem die Adoption vorgenommen wurde, nach der die Annahme entsprechend des Übereinkommens zustande gekommen ist.

5 Internationales Familienrechtsverfahrensgesetz vom 26.1.2005, BGBI I 2005, 162-174.

6 Haager Übereinkommen über den Schutz von Kindern und die Zusammenarbeit auf dem Gebiet der internationalen Adoption (Internationales Adoptionsübereinkommen) vom 29.5.1993.

7 Zum aktuellen Stand der Mitgliedstaaten www.bundesjustizamt.de.

8 Gesetz zur Ausführung des Haager Übereinkommens vom 29.5.1993 über den Schutz von Kindern und die Zusammenarbeit auf dem Gebiet der internationalen Adoptionen, BGBI I 2001, 2950. 